\title{
Multiplexing technology for in vitro diagnosis of pathogens: the key contribution of phosphorus dendrimers
}

\author{
Jean Pierre Majoral ${ }^{1,2,3^{*}}$, Jean Marie François ${ }^{3,4}$, Richard Fabre ${ }^{3}$, Alice Senescau ${ }^{3}$, Serge Mignani ${ }^{5}$ and
} Anne-Marie Caminade ${ }^{1,2}$

\begin{abstract}
After the microbiology based on Pasteur's method and polymerase chain reaction (PCR), the diagnosis company named Dendris has proposed a third-generation of diagnosis enabling the search of a broad range of pathogens with strong sensitivity and specificity. This extraordinary profile was possible thanks to the use of phosphorus dendrimers for which various techniques of deposition on a given support were investigated and described and analyzed in this report.
\end{abstract}

Keywords: phosphorus, dendrimers, diagnosis, DNA arrays, multiplexing technology

\section{INTRODUCTION}

Successful use and reliability of microarrays technology are highly dependent on several factors including surface chemistry parameters and accessibility of cDNA targets to the DNA probes fixed onto the surface. Activated glass slides were commonly used because of their great chemical resistance against solvents, mechanical stability and low intrinsic fluorescence properties. Their performances, i.e., signal intensity, signal-to-noise ratio, spot homogeneity are directly influenced by the amount of DNA probes attached to the surface, the probe length and the accessibility of the labeled targets to the probes $[1,2]$.

While good quality, accuracy and reliability of several microarrays were mentioned, most of them were suffer- ing from the non-linearity of the structure of the spacer which leads to neither a significant increase of the accessibility of the target to the probes nor of the loading capacity of the support [3-6].

To overcome these major drawbacks, dendrimers (monodisperse nanometric spherical structures), which can be tailored at will, were used as linkers. However difficulties encountered for their multistep synthesis, and low thermal stability of some of these nano-objects prevent from their common use for biological applications [7-12].

In marked contrast, the original contribution of stable phosphorus dendrimers as linkers for DNA based multiplexing technology for rapid and accurate diagnosis of pathogens can be emphasized and will be illustrated in this report through the original methodology developed by the Dendris' company.

\section{PHOSPHORUS DENDRIMERS DEDICATED FOR GRAFTING BIOMOLECULES}

The first experiments concerning the design of dendritic bio-arrays starting from phosphorus dendrimers were reported in 1999 using a phosphorus dendrimer of generation 5 built from a trifunctional core and bearing 96 terminal aldehyde groups [13]. This dendrimer was added to quartz or glass slides pretreated by aminopropyltriethoxysilane [14]. Atomic force microscopy (AFM)

${ }^{1}$ Laboratoire de Chimie de Coordination du CNRS, 31077 Toulouse Cedex 4, France

${ }^{2}$ LCC-CNRS Université de Toulouse, CNRS, Toulouse, France

${ }^{3}$ Dendris, 31670 Labège, France

${ }^{4}$ LISBP, UMR CNRS 5504 INRA 792, 31077 Toulouse Cedex 04, France

${ }^{5}$ Université Paris Descartes PRES Sorbonne Paris Cité, CNRS-UMR 860, Laboratoire de Chimie et de Biochimie Pharmacologiques et Toxicolgique, 75006 Paris, France

" Corresponding author (email: jean-pierre.majoral@lcc-toulouse.fr) 


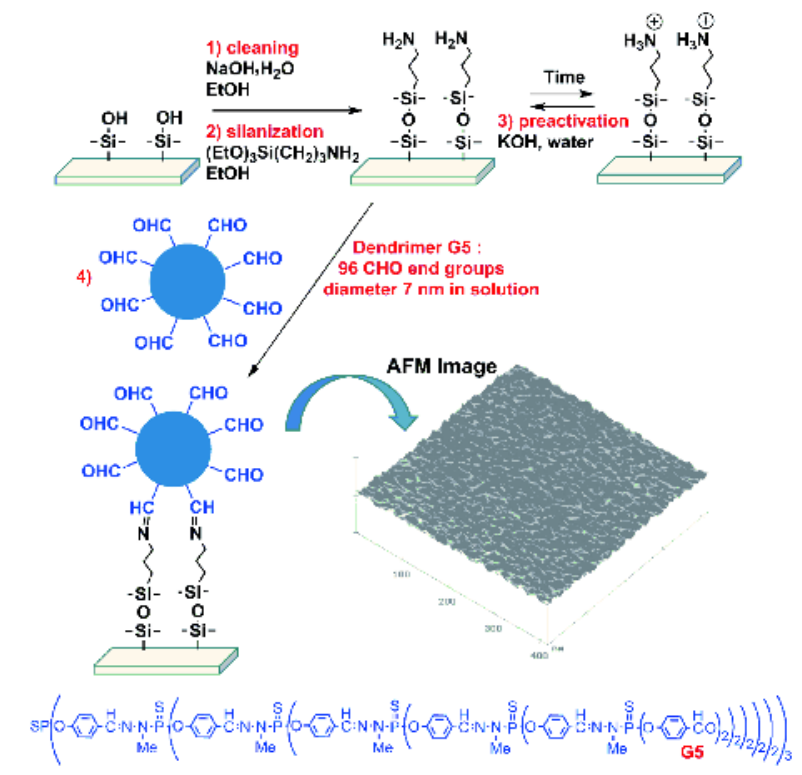

Figure 1 Grafting of aldehyde terminated phosphorus dendrimer on aminosilanized glass slide.

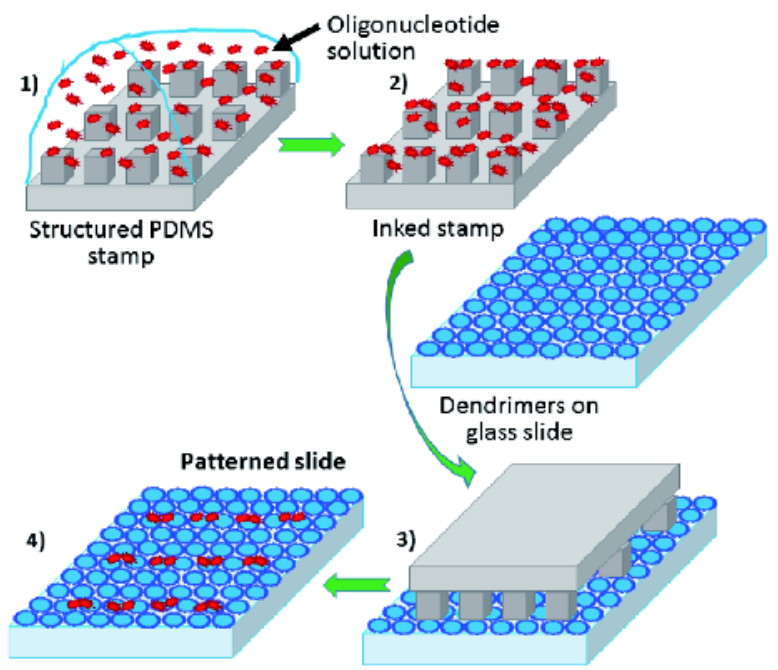

Figure 2 Microcontact printing of DNA involving four steps: 1) inking of the stamp with oligonucleotide, 2) drying under nitrogen stream, 3) contact of the glass slide functionalized by dendrimers with the inked PDMS stamp, 4) transfer of oligonucleotide from the PDMS stamp to obtain the patterned slide.

experiments clearly showed the perfect coverage of the surface of the glass slide (the thickness of the dendrimer layer on the glass slide was found to be $3.7 \mathrm{~nm}$ ) (Fig. 1). The interaction of this surface with proteins bearing $\mathrm{NH}_{2}$ units such as human serum albumin (HSA) was also shown by AFM.

Such a methodology was applied later on for the formation of DNA microarrays involving phosphorus den- drimers obtained from a hexafunctional core and bearing 48 or 96 aldehyde end groups [15], which allow both the direct grafting on aminosilanized glass slides and in a second step the reaction of the remaining aldehyde groups with aminooligonucleotides [16-17].

Grafting to liposomes oligonucleotides complementary to the nucleotides bound to these DNA chips led to nanocapsules arrays potentially usable for the encapsulation and spotting of proteins keeping their conformation and activity within the liposomes [18].

The phosphorus dendrimer of generation 4 decorated on its surface with 96 aldehyde groups can be also linked to a silica piezoelectric membrane. In this particular case the hybridized complementary oligonucleotide does not bear a fluorescent label but a biotin label which can be recognized by streptavidin-conjugated gold nanoparticles. The modification of the resonant frequencies of these functionalized piezoelectric-membranes was measured via an optical beam deflection technique [19].

Another method to create DNA arrays starting from phosphorus dendrimers consists in using stamps for microprinting (Fig. 2). Recent studies reported the use of an automated microcontact printing device using softlithography technology to produce low-density multiplexed DNA microarrays. This automatically allows a multiplexed deposition of oligo probes on a functionalized surface by the use of a macrostamp bearing 64 individual pillars each mounted with 50 circular micropatterns (spots) of $160 \mu \mathrm{m}$ diameter. A fast and efficient washing step in ethanol made this macrostamp reliable and reusable. The low-density microarrays printed on either epoxysilane or phosphorus dendrimer functionalized slides show excellent hybridization responses with complementary sequences at unusually low probe and target concentrations $\left(1 \mu \mathrm{mol} \mathrm{L^{-1 }}\right.$ and $1 \mathrm{nmol} \mathrm{L}^{-1}$, respectively). Comparison of analysis of microarrays obtained from mechanical spotting and automated microprinting method highlights the importance of the accessibility rather than the absolute density of the probes in the hybridization response [20].

A sophisticated highly sensitive DNA hybridization was reported using nanotubes of dendrimers formed inside an ordered porous alumina membrane. The nanotubes were obtained by layer-by-layer (LBL) deposition of negatively and positively charged phosphorus dendrimers on the pore walls. After deposition of three bilayers of anionic and cationic dendrimers, a first layer of negatively charged quantum dots (QDs) was deposited. The alternate deposition of polycationic dendrimers with several other layers of three different luminescent QDs offered a 
graded-bandgap structure. Then probe oligonucleotides were immobilized, and hybridized with target fluorescent labeled oligonucleotides. An efficient excitation energy transfer occurs by fluorescence resonance energy transfer (FRET) from the outer to the inner surface of the dendrimer nanotubes and allowed detection of hybridization with a very high sensitivity: $2-5$ pmol L $^{-1}$ (Fig. 3) [21,22].

Dendrimer multilayers on gold substrates prepared by LBL assembly technique were characterized and used as substrates for DNA immobilization/hybridization. The multilayers were built using alternatively polycationic and polyanionic phosphorus dendrimers of generation 4: a high loading of DNA probes was obtained through covalent coupling of probe DNA on dendrimer multilayers film. The following hybridization of Cy5 dye labeled complementary target DNA with immobilized probe DNA was detected by surface plasmon field-enhanced fluorescence spectroscopy. The limit of detection of target DNA upon hybridization reached $50 \mathrm{pmol} \mathrm{L}^{-1}$ and $30 \mathrm{pmol} \mathrm{L}^{-1}$ on 1 bilayer and 4 bilayers, respectively (Fig. 4) [23].

A bilayer formed with a perylene diimide labelled star polymer donor $\mathbf{A}$ and a cationic phosphorus dendrimer $\mathbf{B}$ (Fig. 5) on a silver substrate was constructed by layer-bylayer approach. Using surface plasmon enhanced fluorescence spectroscopy technique, a time-resolved ultrasensitive $\left(10^{-18} \mathrm{~mol} \mathrm{~L}^{-1}\right)$ and selective detection of DNA targets relying on enhanced optical fields associated with energy transfer were achieved under the excitation at $543 \mathrm{~nm}$. The detection limit is about 8 orders of magnitude better than the achieved one under the excitation at $632 \mathrm{~nm}$, which is ascribed to no energy transfer from the donor to the acceptor under the excitation at $632 \mathrm{~nm}$, resulting in much weaker detection signal in turn (Fig. 6) [24].

A strategy to covalently immobilize biomolecules on AFM tips was also reported on the very classical glutathione-S-transferase (GST)/anti GST protein pair. The AFM tips were first functionalized with amino groups then incubated in a phosphorus dendrimer solution, the dendrimer of generation 4 being decorated with 96 aldehyde groups on its surface. The free aldehyde groups on the surface of the dendrimer are available to react further with amino groups present on every protein and biomolecules. Indeed the dendrimer is acting as a linker between AFM tip and biomolecules. Using these biofunctionalized-dendritips, single molecule force interactions between GST immobilized on a slide functionalized with phosphorus dendrimers (dendrislides), and its cognate antibody immobilized on dendritips were probed by

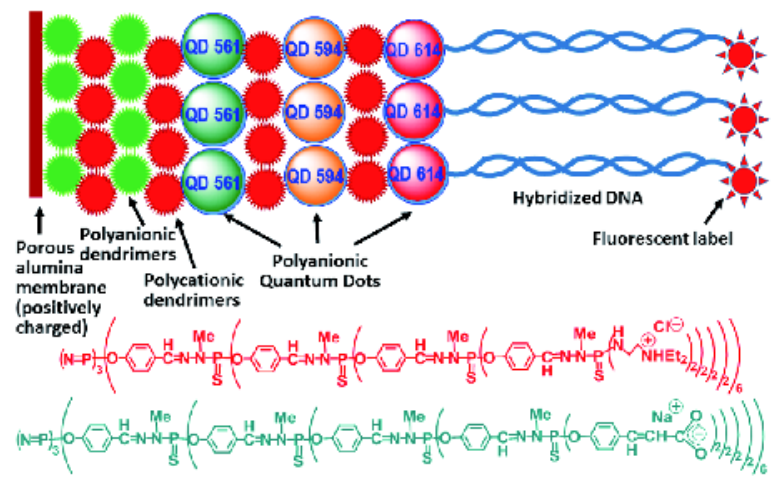

Figure 3 Graded-bandgap structures based on charged phosphorus dendrimers and quantum dots inside an ordered porous alumina membrane.

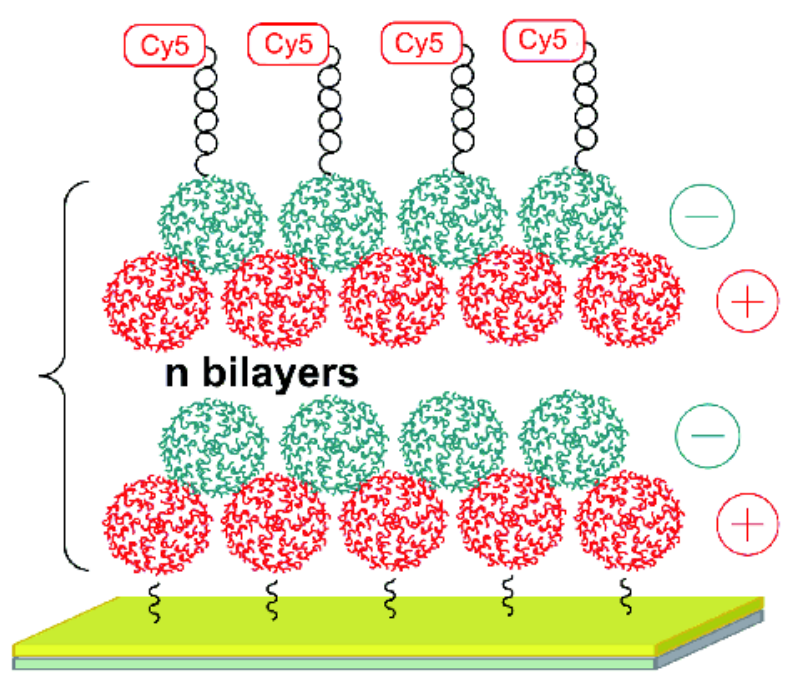

Figure 4 Hybridization of Cy5 complementary target DNA with DNA immobilized on phosphorus dendrimer multilayers (structures of the dendrimers are shown in Fig. 3).

AFM spectroscopy with high specificity [25].

\section{THE DENDRIS WAY}

All the technologies briefly presented up to now, appear of interest but suffer from numerous drawbacks and more precisely they are difficult to extend to for example multiplexing and they are quite expensive. Therefore it was a need to propose cheaper, sensitive and reliable DNA microarrays production.

The strategy developed by Dendris is illustrated in Fig. 7. Covalent attachment of the spherical reactive phosphorus dendrimer of generation 4 bearing 96 terminal aldehyde groups on amino-silanized glass slides generates a reactive $\sim 50 \AA$ layer onto which aminomodified DNA probes are covalently bound. The new 


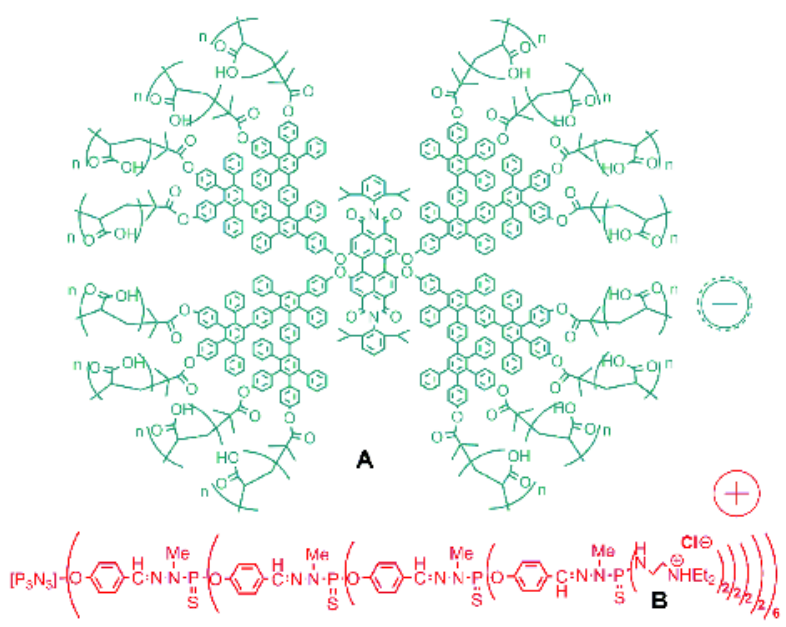

Figure 5 Structure of the anionic perylene diimide labelled star polymer $\mathbf{A}$ and of the cationic phosphorus dendrimer $\mathbf{B}$.

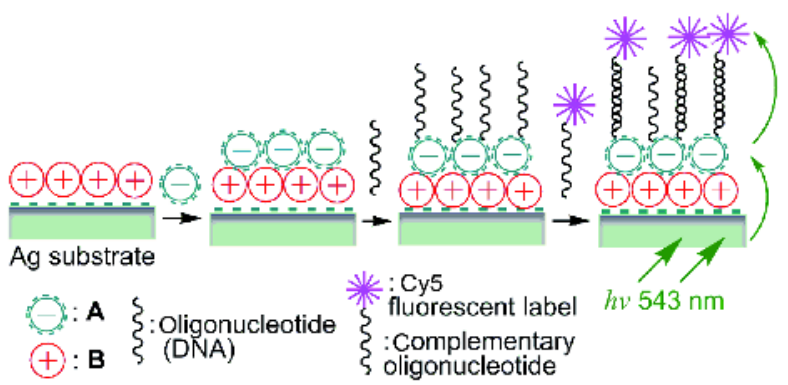

Figure 6 Sensitivity up to $10^{-18}$ obtained from a bilayer formed with $\mathbf{A}$ and $\mathbf{B}$ (Fig. 5) and using surface plasmon enhanced fluorescence spectroscopy technique.

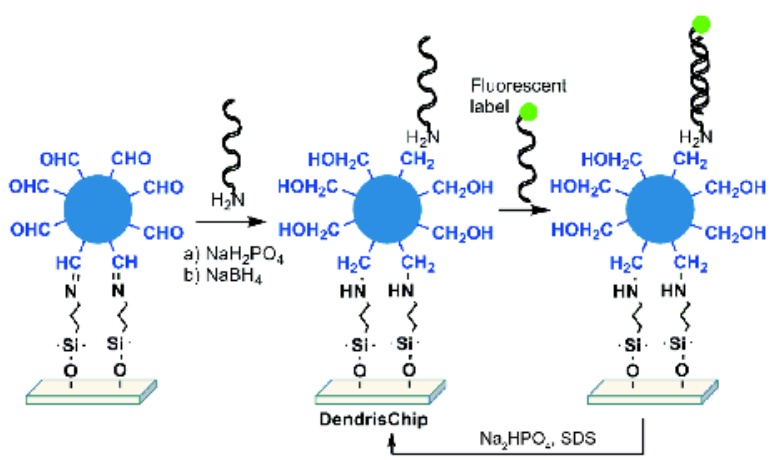

Figure 7 The DNA microarrays technique developed by Dendris.

grafting chemistry leads to the formation of uniform and homogeneous spots. Moreover, probe concentration before spotting could be reduced from 0.2 to $0.02 \mathrm{mg} \mathrm{mL}^{-1}$ with PCR products and from 50 to $5 \mu \mathrm{mol} \mathrm{L} \mathrm{L}^{-1}$ with 25 to 70 mer oligonucleotides without affecting signal in-

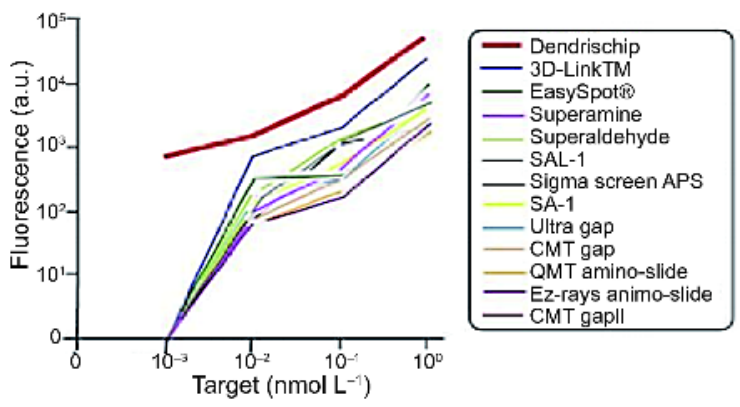

Figure 8 The advantages of DendrisChip compared to other potential competitors. Reprinted with permission from [14].

tensities after hybridization with Cy3- and Cy5-labeled targets. The binding efficiency of these dendrislides was remarkably high, a fluorescent signal being detectable at 1 pmol L $\mathrm{L}^{-1}$ concentration of target that is to say 10 - to 100 fold higher than arrays made with most of other functionalized glass slides. Interestingly reusability of these dendrislides was possible, based on the fact that the signal intensity of DNA target to oligoprobes was not reduced even after 10 hybridization/stripping cycles [17].

The functionalization of the glass slides with phosphorus dendrimers benefits from three advantages: i) an increase of the density of probes per unit surface and thus in the number of binding sites; ii) a greater accessibility of the target to the probes with hybridization in 3D and not 2D like many other activated surfaces; and iii) a greater spacer from the glass surface reducing the unspecific interaction. Fig. 8 focuses on the advantages of DendrisChip compared to other competitors. The interaction between probe and target is 10- to 100-times higher than with the others.

Since a same clinical symptom diagnosed by a physician can be caused by more than one pathogen, the new paradigm is to consider a syndromic approach of the diagnosis, which basically aims at identifying all potential pathogens responsible for the syndrome. This new approach also coincides with the exponential demand of new in vitro diagnosis (IVD) techniques that are more informative by providing more data from a single biological sample, while being more precise, fast and affordable at cost-effective value. The syndrome-based approach of IVD requires a method, leading to the development and commercialization of multiplex PCR technology allowing parallel identification of up to 22 entities, but they all require expensive equipment.

The polymerase chain reaction (PCR), a technique which is used to amplify any DNA or cDNA is, at present, the most powerful tool of molecular diagnosis due to its 
sensitivity and specificity. As the identity of every living species, its molecular characterization, its potential, virulence and pathogenicity, depend on its DNA sequence, the scope of PCR is quite unlimited in human clinical biology, veterinary and plant microbiology. In addition, by avoiding culture, PCR opens the scope of non- or not easily cultivable germs as anaerobic bacteria or viruses

However even if the high specificity related to the very fitted hybridization of the two primers on the targeted DNA is the main advantage to use the PCR, there is a real limitation, as each PCR is unique: one virus, or bacteria is detected at a time. If one wants to detect simultaneously more than one target (multiplex) there are only two ways: enhancing the number of couples of primers to specifically target each of the biological entities, with the risk of interferences, or using the variability inside a given DNA sequences (target) such as the 16S RNA in bacterial genome [26]. The latter one is the most powerful option, but it implies to manage a more complex process able to decipher the slightest differences in the various sequences melted in the postPCR products. Several methods are available, and the simplest one is to use the melting curves produced in the PCR thermocycler by lowering step by step the temperature of the mix from $95^{\circ} \mathrm{C}$ to $40^{\circ} \mathrm{C}$. This melting point is a physical characteristic of the G/C content of the sequence. Sequencing the PCR product is another way to assess precisely its sequence as well as the use of mass spectrometry techniques. These ways are very acute but expensive. Therefore, the Dendris way represents another solution as shown above: the use of DNA chips where post PCR products are hybridized on covalently fixed specific probes of each possible variant of the target.

As we use a piezo electric device to spot probes of $120-150 \mu \mathrm{m}$ diameter size on glass slide, their number can be very high, but the challenge of Dendris is to transform DNA chips in a rapid, simple, robust and easyto-use test able to be performed in ordinary clinical labs. Therefore, in practice, we spot hundred to specifically detect and discriminate biological entities linked to a given symptom or application. The contribution of DNA chip to the process after PCR is its ability of multiplexing, enhancing in the same way the productivity of PCR and opening the microbiology to syndromic diagnostic. Table 1 gives a short overview of the advantages of our molecular technology as compared to multiplex PCR and standard microbiological methods.

Based on our technical specificity of dendrimer chemistry reported above, Dendris has developed an affordable
Table 1 Comparative analysis of criteria from the different technologies used in IVD

\begin{tabular}{ccccc}
\hline & $\begin{array}{c}\text { Culture } \\
\text { method }\end{array}$ & $\begin{array}{c}\text { Mass spec- } \\
\text { trometry }\end{array}$ & PCR & Dendrischip \\
\hline Multiplex & No & No & Yes & Yes \\
Time of response & $48 \mathrm{~h}$ & $24 \mathrm{~h}$ & $1-3 \mathrm{~h}$ & $5 \mathrm{~h}^{\mathrm{a}}$ \\
$\begin{array}{c}\text { Non-cultivable } \\
\text { species }\end{array}$ & No & No & Yes & Yes \\
$\begin{array}{c}\text { Automatization } \\
\text { Man power cost }\end{array}$ & No & Semi & Yes & Yes \\
\hline
\end{tabular}

a) Including PCR amplification

and easy-to-use multiplex diagnosis, for syndrome-based diseases and biomarkers validation. The kit is intended for hospitals and medical laboratories with a user-friendly IVD device. In a single test, it provides a rapid and accurate diagnosis, thus improving and accelerating the medical decision making. The IVD solution shall lead to a new standard, which could reduce public health costs. Rapid diagnosis will establish a faster curative action, and determine the best treatment to prescribe in the context of personalized medicine. The $\beta$-tester prototype available today from Dendris is depicted in Fig. 9. It includes a dedicated scanner (Dendriscan) to read the DendrisChip. In addition, it contains a simple SoftDiag for data analysis which is based on an algorithmic method.

The statistical method allows to classify and discriminate most of the bacteria species used in the training. Thus the DendrisChip provides an accurate diagnostic and moreover allows questioning about identification assessed by microbial methods.

\section{EXAMPLE OF APPLICATIONS OF DENDRISCHIPS}

Our first application has been dedicated to respiratory

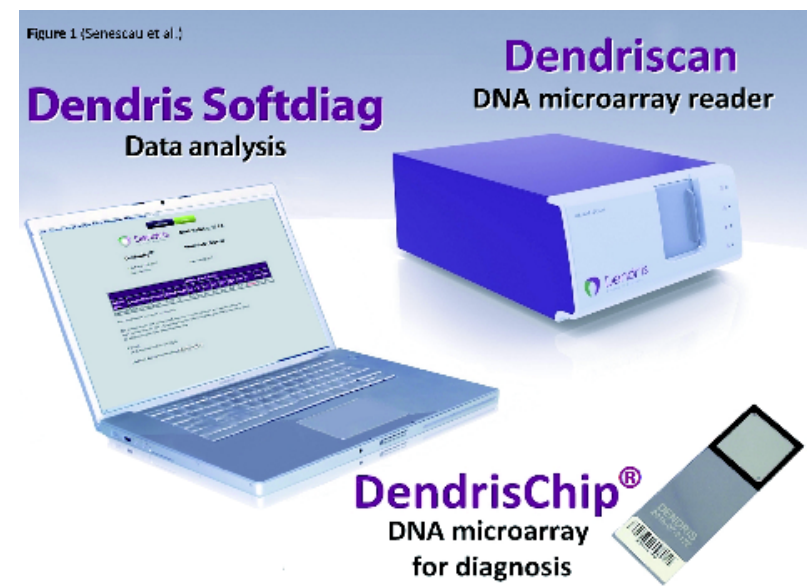

Figure $9 \beta$-tester prototype proposed by Dendris. 
tract infection which is the most widespread infectious disease, and is caused by a wide range of micro-organisms which are difficult to clinically distinguish due to symptoms similarities. The symptom approach developed thanks to the DendrisChips technology allows rapidly to discriminate 11 bacteria: Streptococcus pneumoniae (Spn), Haemophilus influenzae (Hi), Neisseria meningitidis $(\mathrm{Nm})$, Moraxella catharralis (Mca), Chlamydiae pneumoniae (Cpn), Mycoplasma pneumoniae (Mpn), Legionella pneumophila (Lpn), Klebsiella pneumoniae (Kpn), Pseudomonas aeruginosa (Pa), Staphylococcus aureus (Sau) and Bordetella pertussis (Bp), responsible for this infection disease.

Our technology has been compared with the still currently largely used microbiological culture. We showed a detection limit in the range of $10^{3}$ cell $\mathrm{mL}^{-1}$ which is in the same range as the culture methods. In addition, the reliability of our DendrisChips technology in the detection of pathogen bacteria was challenged over more than 250 clinical specimens and the results were compared with culture methods. In numerous cases the DendrisChip confirmed the presence of a pathogen suspected but not confirmed by culture in several examples and revealed more information thanks to its ability to detect co-infections. To further evaluate the accuracy of our molecular method, we applied a sensitivity criterion which is defined as the ratio between the number of times a given pathogen has been detected over the total number of sample in which this pathogen was present (i.e., detected by both DendrisChip and microbiological method). This comparative analysis is reported in Fig. 10. Results showed a better sensitivity criterion of DendrisChip than culture method notably for $H$. influenzae (95\% vs. 62\%) and for P. aeruginosa (94\% vs. $82 \%)$. High sensitivity for K. pneumoniae and M. catharralis was also obtained with our technology but these values must be taken with caution owing to the low number of clinical samples containing these pathogens. On the other hand, the sensitivity criterion of our technology was apparently very low with respect to $S$. aureus (66 \% vs. 94\%). However, we re-evaluated the microbiological result using the specific PCR assay for this pathogen developed by $\mathrm{BD}$ diagnostic and found that only 10 out of the 31 samples positively identified by the microbiological culture were confirmed by the BDmax PCR assay. Therefore, one can conclude that even with $S$. aureus, our technology was as accurate as the culture method. Finally, B. pertussis, L. pneumophila, N. meningitidis, Chlamydiae pneumoniae or $M$. pneumoniae were never found by microbiological culture in the 238 clinical specimens.

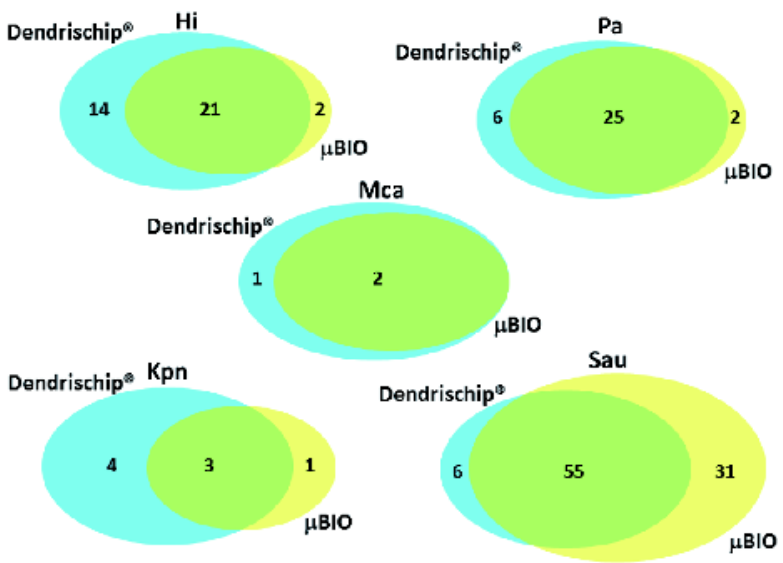

Figure 10 Sensitivity of the DendrisChips technology (sum of blue and green ovals) versus microbiological method (sum of yellow and green ovals). Hi: H. influenzae; Pa: P. aeruginosa; Mca: M. catharralis; Kpn: $K$. pneumoniae; Sau: S. aureus. Caution : in the case of Sau, only 10 out of the 31 samples positively identified by the microbiological culture were confirmed by BDmax PCR assays.

The Dendris solution of IVD in respiratory infection is fully compliant with medical practice. This method shall not solely complete traditional diagnostic assays, but overcome limitation of these current assays in providing a rapid, accurate and more informative diagnostic, which could reduce the use of antibiotic therapies.

Other applications that are currently in development by Dendris are in the following area:

Clinical microbiology: According to the World Health Organization (WHO), more than 1 million sexually transmitted infections (STIs) are acquired every day worldwide. Each year, there are an estimated 357 million new infections with 1 of 4 STIs: chlamydia, gonorrhoea, syphilis and trichomoniasis and more than 500 million people are estimated to have genital infection with herpes simplex virus (HSV). The majority of STIs have no symptoms or only mild symptoms that may not be recognized as an STI. In some cases, STIs can have serious reproductive health consequences beyond the immediate impact of the infection itself (e.g., infertility or mother-tochild transmission). Dendris develops a DendrisChip dedicated to the detection of 10 bacteria, virus or parasites known to be transmitted through sexual contact: Candida albicans, Chlamydiae trachomatis, Haemophilus ducreyi, HSV1, HSV2, Mycoplasma genitalium, Neisseria gonorrhoeae, Streptococcus agalactiae, Trichomonas vaginalis and Treponema pallidum. This application is currently in development and will be available in 2019 .

Agro-nutrition and food safety: The search of contamination in alimentary stuffs and breeding, will follow 
the same way than clinical microbiology due to the impact on human welfare; but due to the heavy economic constraints in this case evolution will be slower. Thanks to a partnership with academical and analysis laboratories, we have worked on the design of a DendrisChip able to detect the most important bacteria in food safety (Staphylococcus aureus, Listeria monocytogenes, Yersinia enterolytica, Bacillus cereus, Salmonella spp., and 6 serotypes of E. coli). This DendrisChip was validated on bacterial strains and tests on food samples are in progress.

Environment microbiology: Dendris is aiming to provide an easy to use method to detect and discriminate legionella species that contaminate water cooling towers. Our actual pre-prototype is actually able to discriminate at least 21 different Legionella species among which the four pathogens, namely L. pneumophila, L. anisa, L. bozemanii and L. longbeachae

Medical genomics: To identify some markers of acquired or hereditary diseases on a gene, it is necessary to look for the presence of several mutations. It is therefore useful to characterize precisely these mutations by sequencing but, once they are well-known it is easier and less expensive to use DNA chips, best fitted to decipher mixed alleles for instance on biological samples and tumors. So, they are well fitted to the characterization of tumors as for the diagnosis than for the prognosis or the therapeutic choices. The high costs of the new anticancer molecules and the high specificity of their molecular targets command to decipher the good-responders patients from the non-responders. They are well-known as the companion test.

\section{CONCLUSIONS}

Today, more than $70 \%$ of clinical decisions are based on IVD tests. IVD helps physician to provide the therapeutic and the good management of the patient health. With this decisive role in healthcare, IVD innovation is foreseen to better contribute to health savings and meanwhile is a very important economic sector. That is why many companies keep on investing an important part of their revenue (10 to $12 \%$ ) in IVD R\&D. To address the clinician needs in combination with greater health concern of the patients, the most suitable solutions in IVD shall meet these specific features: i) multiplex rather than simplex (i.e., identify pathogen through a syndromic approach rather than actual mode of single identification by phenotypic or genotypic method), ii) high sensitivity, iii) high specificity, iv) acceptable costs of the whole process (reagents, instrument, and man power), v) non-invasive way.
DNA chips may be the very promising multiplexing tool the biology has a huge and urgent need. DNA chips must progress in the scope of cost, robustness and mainly automation. They have the potential, but they need a large cooperation between biologists, chemists, computer scientists and engineers. The great enhancement performed in that way by Dendris in clinical DNA chips by using phosphorus dendrimers as surface chemistry is a big step forward. In 2018, the main part of medical bacteriology depends on Petri dishes invented in 1887. By paving the way to syndromic diagnosis, to the characterization of non- or ill-cultivable germs and by saving time offered to the clinicians for the right treatment of their patients, the DNA chips post PCR will contribute to the fall of Petri dishes in less than the next ten years. To be a substitute to a centennial process is what we call a disruption.

The solution proposed by Dendris based on the use of phosphorus dendrimers fits perfectly with all requirements summarized above, and forward-looking.

Received 14 February 2018; accepted 17 March 2018;

published online 16 April 2018

1 He Z. Microarrays: Current Technology, Innovations, and Applications. Poole: Caister Academic Press, 2014

2 Jo $\mathrm{H}$, Lee $\mathrm{S}$, Ban C. Highly sensitive and selective in vitro diagnostics based on DNA probes and aptamers. Biodesign, 2015, 3: $33-40$

3 Joos B, Kuster H, Cone R. Covalent attachment of hybridizable oligonucleotides to glass supports. Anal Biochem, 1997, 247: 96101

4 Rogers YH, Jiang-Baucom P, Huang ZJ, et al. Immobilization of oligonucleotides onto a glass support via disulfide bonds: a method for preparation of DNA microarrays. Anal Biochem, 1999, 266: 23-30

5 Donatin E, Drancourt M. DNA microarrays for the diagnosis of infectious diseases. Médecine Maladies Infectieuses, 2012, 42: 453459

6 Pillet S, Lardeux M, Dina J, et al. Comparative evaluation of six commercialized multiplex PCR kits for the diagnosis of respiratory infections. PLoS ONE, 2013, 8: e72174

7 Benters R, Niemeyer CM, Wöhrle D. Dendrimer-activated solid supports for nucleic acid and protein microarrays. ChemBioChem, 2001, 2: 686-694

8 Tomalia DA, Naylor AM, Goddard WA. Starburst dendrimers: molecular-level control of size, shape, surface chemistry, topology, and flexibility from atoms to macroscopic matter. Angew Chem Int Ed Engl, 1990, 29: 138-175

9 Park JW, Jung Y, Jung YH, Seo JS, Lee Y. Preparation of oligonucleotide arrays with high-density DNA deposition and high hybridization efficiency. Bull Korean Chem Soc, 2004, 25: 16671670

10 Benters R. DNA microarrays with PAMAM dendritic linker systems. Nucleic Acids Res, 2002, 30: 10e-10

11 Ahmed S, Vepuri SB, Kalhapure RS, et al. Interactions of den- 
drimers with biological drug targets: reality or mystery-a gap in drug delivery and development research. Biomater Sci, 2016, 4: 1032-1050

12 Svenson S. The dendrimer paradox-high medical expectations but poor clinical translation. Chem Soc Rev, 2015, 44: 4131-4144

13 Launay N, Caminade AM, Majoral JP. Synthesis and reactivity of unusual phosphorus dendrimers. a useful divergent growth approach up to the seventh generation. J Am Chem Soc, 1995, 117: 3282-3283

14 Slomkowski S, Miksa B, Chehimi MM, et al. Inorganic-organic systems with tailored properties controlled on molecular, macromolecular and microscopic level. Reactive Funct Polymers, 1999, 41: $45-57$

15 Launay N, Caminade AM, Majoral JP. Synthesis of bowl-shaped dendrimers from generation 1 to generation 8. J Organomet Chem, 1997, 529: 51-58

16 Le Berre V. Dendrimeric coating of glass slides for sensitive DNA microarrays analysis. Nucleic Acids Res, 2003, 31: 88e-88

17 Trévisiol E, Le Berre-Anton V, Leclaire J, et al. Dendrislides, dendrichips: a simple chemical functionalization of glass slides with phosphorus dendrimers as an effective means for the preparation of biochips. New J Chem, 2003, 27: 1713-1719

18 Chaize B, Nguyen M, Ruysschaert T, et al. Microstructured liposome array. Bioconjugate Chem, 2006, 17: 245-247

19 Nicu L, Guirardel M, Chambosse F, et al. Resonating piezoelectric membranes for microelectromechanically based bioassay: detection of streptavidin-gold nanoparticles interaction with biotinylated DNA. Sensor Actuat B-Chem, 2005, 110: 125-136

20 Thibault C, Le Berre V, Casimirius S, et al. Direct microcontact printing of oligonucleotides for biochip applications.. J Nanobiotechnol, 2005, 3: 7

21 Feng CL, Zhong X , Steinhart M, et al. Graded-bandgap quantum- dot-modified nanotubes: a sensitive biosensor for enhanced detection of DNA hybridization. Adv Mater, 2007, 19: 1933-1936

22 Feng CL, Zhong XH, Steinhart M, et al. Functional quantum-dot/ dendrimer nanotubes for sensitive detection of DNA hybridization. Small, 2008, 4: 566-571

23 Yu Y, Feng C, Caminade AM, et al. The detection of DNA hybridization on phosphorus dendrimer multilayer films by surface plasmon field enhanced-fluorescence spectroscopy. Langmuir, 2009, 25: $13680-13684$

24 Feng CL, Yin M, Zhang D, et al. Fluorescent core-shell star polymers based bioassays for ultrasensitive DNA detection by surface plasmon fluorescence spectroscopy. Macromol Rapid Commun, 2011, 32: 679-683

25 Jauvert E, Dague E, Séverac M, et al. Probing single molecule interactions by AFM using bio-functionalized dendritips. Senss Actuators B-Chem, 2012, 168: 436-441

26 Patel JB. 16S rRNA gene sequencing for bacterial pathogen identification in the clinical laboratory. Mol Diagnosis, 2001, 6: 313321

Acknowledgements This work was supported by the National Research Agency (Agence Nationale pour la Recherche), "BIOTECHNOLOGIES" program (ANR 2010 BIOT 004 06: Project INNODIAG to JMF) and by Region Midi Pyrénées (06001324 \& 07006292) to RF and JMF and by CNRS (JPM, AMC).

Author contributions Majoral JP, Caminade AM designed experiments linked to dendrimer technology, François JM and Fabre R conceived the DendriSchip kit fabrication. Majoral JP wrote the paper which was improved and approved by all the other authors.

Conflict of interest The authors declare no conflict of interest.

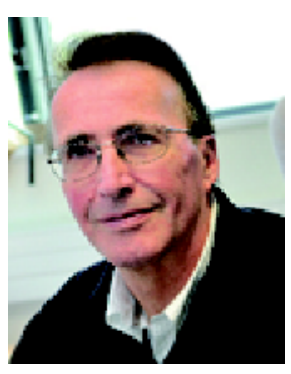

Jean-Pierre Majoral is Emeritus Director of Research, Exceptional Class at the CNRS in Toulouse. His research interest is focused on the design and the properties of macromolecules such as phosphorus dendrimers and hyperbranched polymers. Main efforts are directed at the use of dendrimers in medicinal chemistry, material sciences and catalysis. He is cofounder and scientific director of the start-up Dendris. He is a member of several Academies of Sciences worldwide, got a dozen of international awards, and is an author of over 635 publications, 7 books, 35 book chapters, and 45 patents (h index 65 , over 15,700 citations). 